\title{
Mapeamento da publicação científica da Fonoaudiologia brasileira na área voz do professor: um protocolo de revisão de escopo
}

\author{
Mapping the scientific publication of Brazilian speech therapy in the area of the teacher's voice: a \\ scope review protocol
}

Mapeo de la publicación científica de la fonoaudiología brasileña en el área de la voz del docente: un protocolo de revisión de alcance

\author{
Recebido: 29/07/2021 | Revisado: 04/08/2021 | Aceito: 06/08/2021 | Publicado: 12/08/2021 \\ Edna Pereira Gomes de Morais \\ ORCID: https://orcid.org/0000-0002-0034-0166 \\ Universidade Estadual de Ciências da Saúde de Alagoas, Brasil \\ E-mail: edna.gomes@uncisal.edu.br \\ Marcelo de Araújo Santos \\ ORCID: https://orcid.org/0000-0003-4442-7826 \\ Universidade Estadual de Ciências da Saúde de Alagoas, Brasil \\ E-mail: marceloaraujosssantos@gmail.com \\ Lavinia Vieira Dias Cardoso \\ ORCID: https://orcid.org/0000-0002-0179-8523 \\ Universidade Estadual de Ciências da Saúde de Alagoas, Brasil \\ E-mail: laviniacardoso.fono@gmail.com \\ Vanessa Fernandes de Almeida Porto \\ ORCID: https://orcid.org/0000-0002-0100-4869 \\ Universidade Estadual de Ciências da Saúde de Alagoas, Brasil \\ E-mail: vanessa.porto@uncisal.edu.br
}

\begin{abstract}
Resumo
Objetivo: mapear e caracterizar as pesquisas na área da voz do professor, publicados em periódicos nacionais de Fonoaudiologia, no período de 2011 a março de 2021, por meio de uma revisão de escopo. Método: será desenvolvida uma estratégia de busca em três passos e as bases de dados utilizadas serão LILACS e SciELO, que indexam os periódicos de Fonoaudiologia. Serão considerados apenas artigos científicos, publicados entre janeiro de 2011 a março de 2021, no idioma português, envolvendo pesquisas primárias com professores, dos diversos níveis de ensino, carga horária, tempo de profissão e de instituições públicas ou privadas. Uma busca nas referências dos estudos selecionados e nos sites dos periódicos serão complementares. A extração dos dados será realizada por meio de um formulário elaborado para a presente revisão e os resultados apresentados em forma de gráficos e tabelas, acompanhados de uma narrativa. Resultados esperados: A diversidade de estudos e a busca pela compreensão da distribuição destes, ao longo dos anos, na Fonoaudiologia brasileira, permitirá um conhecimento amplo sobre como tem sido conduzidas as pesquisas na área. Considerações finais: Compreender as pesquisas desenvolvidas, por meio do mapeamento aqui proposto, e publicizar seus resultados, permitirá aos profissionais que trabalham na área da voz do professor um conhecimento do cenário atualizado. O que permitirá a projeção de estudos futuros e uma prática apoiada em evidências.
\end{abstract}

Palavras-chave: Professores; Disfonia; Distúrbios da fonação; Distúrbios da voz.

\begin{abstract}
Objective: The objective of the present study is to map and characterize research in the field of the teacher's voice, published in national journals of Speech Therapy, in the period from 2011 to March 2021, through a scope review. Method: a three-step search strategy will be developed and the databases used will be LILACS and SciELO, which index the Speech Therapy journals. Only scientific articles will be considered, published between January 2011 and March 2021, in Portuguese, involving primary research with teachers, from different levels of education, workload, time of profession and from public or private institutions. A search on the references of the selected studies and on the websites of the journals will be complementary. Data extraction will be performed using a form prepared for this review and the results presented in the form of graphs and tables, accompanied by a narrative. Expected results: The diversity of studies and the search for understanding the distribution of these, over the years, in Brazilian Speech Therapy, will allow a broad knowledge of how research has been conducted in the area. Final considerations: Understanding the research developed, through the mapping proposed here, and publicizing its results, will allow
\end{abstract}


professionals who work in the area of the teacher's voice to gain knowledge of the updated scenario. This will allow the projection of future studies and evidence-based practice.

Keywords: Teachers; Dysphonia; Phonation disorders; Voice disorders.

\begin{abstract}
Resumen
Objetivo: mapear y caracterizar investigaciones en el área de la voz del docente, publicadas en revistas nacionales de Fonoaudiología, desde 2011 hasta marzo de 2021, a través de una revisión de alcance. Método: se desarrollará una estrategia de búsqueda de tres pasos y las bases de datos utilizadas serán LILACS y SciELO, que indexan las revistas de patología del habla y el lenguaje. Solo se considerarán artículos científicos, publicados entre enero de 2011 y marzo de 2021, en portugués, que involucren investigación primaria con docentes, de diferentes niveles de educación, carga de trabajo, tiempo en la profesión y de instituciones públicas o privadas. Será complementaria una búsqueda en las referencias de los estudios seleccionados y en las webs de las revistas. La extracción de datos se realizará mediante un formulario diseñado para esta revisión y los resultados se presentarán en gráficos y tablas, acompañados de una narrativa. Resultados esperados: La diversidad de estudios y la búsqueda de comprender la distribución de estos, a lo largo de los años, en la Logopedia brasileña, permitirá un conocimiento amplio de cómo se ha realizado la investigación en el área. Consideraciones finales: Comprender la investigación desarrollada, a través del mapeo aquí propuesto, y dar a conocer sus resultados, permitirá a los profesionales que trabajan en el área de la voz del docente conocer el escenario actualizado. Esto permitirá la proyección de futuros estudios y prácticas basadas en evidencias.
\end{abstract}

Palabras clave: Docentes; Afonía; Trastornos de la fonación; Trastornos de la voz.

\title{
1. Introdução
}

As disfonias são consideradas alterações vocais importantes, marcadas pela alteração na produção da voz que prejudica tanto a comunicação social quanto a profissional, sendo os profissionais da voz os mais suscetíveis a esse tipo de alteração (Behlau, Azevedo, \& Pontes, 2001; Ferreira et al., 2016; Schwartz et al., 2009).

De acordo com estudos realizados, os professores apresentam uma prevalência maior quanto à disfonia quando comparados a não professores, numa correspondência de 11,6\% para 7,5\%, respectivamente (Behlau et al., 2012). Um estudo realizado com 317 professores do ensino fundamental e médio, apontou que $81 \%$ dos participantes apresentavam disfonia (Valente et al., 2015).

Os professores são, então, a categoria profissional com maior risco para disfonia e a mais estudada na literatura fonoaudiológica, na área da voz, o que mostra a importância de conhecer mais sobre esse grupo de trabalhadores e os seus aspectos vocais. Entre as pesquisas existentes, há estudos que compreendem temáticas como autopercepção, levantamento de sintomas, avaliação vocal e conhecimento do ambiente de trabalho e suas influências sobre a saúde da voz (Dragone et al., 2010; Morais, 2012).

Para Dragone et al. (2010), o grande número de docentes no Brasil e a acessibilidade a essa população, pode ser o motivo da grande produção dos estudos. Tal aspecto torna-se também um motivador para busca maior da compreensão da disfonia nesse grupo e elaboração de estratégias de intervenção mais seguras e eficazes.

Voltar a atenção para a saúde vocal do trabalhador docente, permite conhecer mais de perto sua problemática e propor ações que venham alertar para os riscos de disfonia, identificando uma possível alteração diante dos primeiros sinais/sintomas, além de permitir uma melhor qualidade de vida, bem-estar vocal e performance durante as aulas, buscando diminuir o problema e os afastamentos de suas atividades laborais. A literatura sobre o tema aponta que os afastamentos e a incapacidade para o desempenho da função docente, implicam em custos financeiros, sociais e até mesmo emocionais (Giannini et al., 2012).

De caráter multifatorial, esses distúrbios ocorrem, na maioria das vezes, devido às más condições de trabalho, além da excessiva demanda de horas-aulas semanais e da elevada intensidade da voz diante da acústica inadequada nas salas de aula e ruídos de intensidade forte (Caporossi \& Ferreira, 2010; Fillis et al., 2016; Gomes et al., 2016).

Mas, apesar dos inúmeros trabalhos nacionais relacionadas a voz do professor, ainda há aspectos a serem explorados, 
principalmente quanto a necessidade de mais estudos experimentais comprovando evidências quanto às intervenções realizadas (Vieira, 2009). Bem como outras temáticas, como aquelas voltadas para os aspectos comunicativos, envolvendo as competências para tal (Araújo et al., 2015; Mortimer, 2014; Ramos et al., 2018; Santos \& Andrada e Silva, 2016). A maioria dos estudos ainda são observacionais descritivos e sobre avaliações, como mostra um estudo anterior, que levantou a publicação sobre o tema na área nos últimos 15 anos, compreendendo o período entre 1994 e 2008. As autoras constaram que a temática avaliação ainda é a mais frequente entre as pesquisas, com 83\% de um total de 500 publicações. Os estudos de intervenção compõem um total de 14\% dos 500 estudos levantados (Dragone et al., 2010).

Ao serem considerados os dados destacados anteriormente, é vista a importância de se conhecer o que já vem sendo explorado na literatura sobre o tema e, assim, refletir sobre propostas futuras de estudos voltados à problemática vocal do professor. Estudos esses que devem abordar a temática desde o ponto de vista para promoção à saúde e bem-estar vocal até o processo de reabilitação, com estudos bem desenhados metodologicamente e capazes de apresentar evidências quanto às intervenções propostas e redução do quadro de adoecimento nessa classe trabalhadora.

Em uma busca preliminar nas bases de dados LILACS (via BVS), Medline (via PubMed), JBI Database of systematic reviews and implementaton reports, The Cochrane database of systematic reviews e OSF Registries, foi encontrada apenas a publicação de um protocolo revisão de escopo (Evangelista et al., 2019), o qual aborda o tema relacionado à promoção da saúde ocupacional com professores de ensino superior. Tal achado mostra a necessidade de se fazer uma revisão mais ampla, até como forma de atualizar a temática, buscando caracterizar os estudos (artigos científicos de pesquisas primárias) sobre do tema "voz do professor", envolvendo todos os níveis de ensino.

Assim, apesar da existência de um protocolo recente, o estudo de revisão de escopo aqui proposto, busca um mapeamento mais amplo, com a finalidade de caracterizar e analisar como as pesquisas estão sendo realizadas com professores de diversos níveis de ensino, quais áreas temáticas vêm sendo exploradas e o que há de lacunas nessa área do conhecimento que precisam ser preenchidas.

Conforme propõem (Arksey \& O’Malley, 2005), por meio de uma revisão de escopo é possível mapear, seguindo um método rigoroso e transparente, o estado da arte de uma determinada área temática, e analisá-los descritivamente, podendo ser um estudo prévio para a condução de outros tipos de estudos, como uma revisão sistemática ou um estudo primário.

Diante do exposto, o objetivo do presente estudo consiste em mapear e caracterizar as pesquisas na área da voz do professor, publicados em periódicos nacionais de Fonoaudiologia, no período de 2011 a março de 2021, por meio de uma revisão de escopo.

\section{Metodologia}

Trata-se de um protocolo de revisão de escopo elaborado segundo as recomendações contidas no Joanna Briggs Institute Reviewer's Manual (Peters et al., 2020) e checklist PRISMA Extension for Scoping Reviews (PRISMA-ScR) (Tricco et al., 2018). Como recomendação inicial, uma pergunta de pesquisa é necessária para nortear a realização da revisão de escopo, seguindo o acrônimo PCC ( $\mathrm{P}$ - participante, C - conceito e C - contexto), o que levou a elaboração da seguinte pergunta de pesquisa: Como estão caracterizadas as pesquisas primárias sobre voz do professor publicadas em periódicos nacionais de Fonoaudiologia, no período de 2011 a março de 2021 ? O registro do protocolo foi previamente realizado na OSF Registration, sob identificação osf.io/7uf8d. 


\subsection{Critérios de Elegibilidade}

Com base na estrutura PCC, os critérios de elegibilidade para o estudo a ser desenvolvido obedeceu ao descrito a seguir:

- Participantes: professores de qualquer nível de ensino (Educação infantil, Ensino Fundamental, Médio ou Superior, além de Educação de Jovens e Adultos), em qualquer faixa etária e gênero, independe do tempo de atuação e serviço (Público ou Privado), que apresentem ou não queixas relacionadas à voz.

- Conceito: serão incluídos estudos primários, na área de voz, realizados com professores de qualquer nível de ensino com presença ou não de disfonia, envolvendo intervenções ou observações, ou mesmo relato de casos ou de experiência, com abordagens que vão desde promoção à saúde ao processo de reabilitação.

- Contexto: periódicos nacionais de Fonoaudiologia que abrigam estudos realizados em qualquer região brasileira, com professores de todos os níveis de ensino.

- Tipos de estudos: serão incluídos artigos científicos de estudos experimentais, quase-experimentais, observacionais analíticos ou descritivos, com abordagem quantitativa, qualitativa (independente do desenho do estudo) ou mista, publicados entre janeiro de 2011 e março de 2021, disponíveis no idioma português.

\subsection{Estratégia de busca}

A estratégia de busca será desenvolvida seguindo três passos, como proposto pelo Joanna Briggs Institute Reviewer's Manual (Peters et al., 2020). Uma busca inicial será realizada no LILACS (via BVS) para identificar e analisar as palavras contidas no título e resumo dos artigos utilizadas para descrevê-los e que poderão ser utilizadas como termos de busca adicionados aos descritores indexados (DeCS). A segunda etapa, mais detalhada, consistirá em uma busca utilizando os descritores e termos identificados na primeira etapa da busca, nas bases de dados LILACS (via BVS) e SciELO, que indexam os periódicos nacionais em Fonoaudiologia. A estratégia de busca será elaborada considerando, no momento, os termos: "professor", "professora", "professores", "professoras", “docente", "docentes", "voz", "disfonia", "distúrbios da voz", "alterações vocais", e serão adaptados para cada base de dados. O terceiro passo consistirá em realizar uma pesquisa adicional nas referências dos artigos que serão incluídos na revisão e nos sites de cada periódico.

Apenas artigos científicos serão considerados, uma vez que se apresentam como a melhor forma de evidências para a proposta da revisão. Também serão impostos limites quanto ao período de publicação, de 2011 à março de 2020, devido ao fato de que busca-se uma atualização desde a última revisão bibliográfica realizada e publicada em 2010, não sendo identificada como revisão de escopo em seu desenho, porém, com uma lacuna de dois anos dos estudos incluídos no estudo de Dragone et al. (2010), pelo fato do estudo ter sido publicado em 2010 e para que pudesse ser levantada as atualizações relacionadas a última década de produção científica sobre o tema. Por se tratar de um estudo que busca mapear a produção nacional brasileira, o idioma "português" foi o eleito.

Nos casos em que um estudo, considerado elegível para inclusão, apresente ausência de dados relevantes para análise, seus autores serão contatados para obtenção de informações adicionais.

Serão excluídos os estudos primários que foram realizados com professores de outras modalidades que não as da Educação infantil, Ensino fundamental, médio, superior e educação de jovens e adultos (EJA).

O Quadro 1 apresenta o modelo da estratégia de busca a ser utilizado para a revisão proposta, trazendo os descritores/palavras-chaves e as combinações por meio dos operadores booleanos a serem utilizados na construção da estratégia de busca. 
Quadro 1. Estratégia de busca elaborada para a presente revisão de escopo;

\begin{tabular}{|l|l|}
\hline \multicolumn{1}{|c|}{ Base } & \multicolumn{1}{|c|}{ Estratégia } \\
\hline & (("professor" OR "professores" OR "docente" OR "docentes") AND ("voz" OR "distúrbios da \\
vILACS (via BVS) & $\begin{array}{l}\text { voz" OR "disfonia")) AND (db:("LILACS") AND la:("pt") AND year_cluster:("2011" OR } \\
\text { "2012" OR "2015" OR "2014" OR "2016" OR "2017" OR "2019" OR "2013" OR "2020" OR }\end{array}$ \\
\hline SciELO & $\begin{array}{l}\text { ("professor" OR "professores" OR "docente" OR "docentes") AND ("voz" OR "distúrbios da } \\
\text { voz" OR "disfonia") }\end{array}$ \\
\hline
\end{tabular}

Fonte: Autores.

\subsection{Seleção dos estudos}

Após o levantamento dos registros, esses serão importados para o gerenciador de referências Mendeley (da Elsevier) e os registros duplicados serão removidos. A seleção dos estudos será realizada por dois revisores (MA; EM) em dois estágios, cujo primeiro consiste na leitura de títulos e resumos e; o segundo estágio, consistirá na leitura dos estudos na íntegra, tomando como base os critérios de inclusão estabelecidos. Os autores farão a leitura de forma independente e, no caso de desacordos, uma terceira autora (VP) definirá pela inclusão ou não do estudo, em ambas as fases da seleção.

As razões para exclusão dos artigos que não atenderam os critérios de inclusão serão registradas e relatadas no escopo da revisão.

As autoras apresentarão os resultados das buscas por meio do Study Flow diagram conforme proposto no PRISMA Extension for Scoping Reviews (PRISMA-ScR) (Tricco et al., 2018) e seguindo as orientações contidas no The PRISMA 2020 statement: an updated guideline for reporting systematic reviews (Page et al., 2021).

\subsection{Extração dos dados}

Para os estudos elegíveis, dois revisores (MA; LC) farão a extração das informações, de forma independente, para um formulário de extração elaborado para a presente revisão (Quadro 2). O formulário conterá informações quanto a população, conceito e contexto, além do desenho de estudo, objetivos, local, temática abordada e principais conclusões. Considerando que o mapeamento é um processo interativo e dinâmico, algumas das informações poderão ser alteradas, ou até mesmo adicionadas. As discrepâncias serão resolvidas por consenso e, caso necessário, uma terceira revisora (VP) poderá arbitrar.

Será realizada uma calibração do formulário, teste piloto, por duas das autoras (EM; VP) de cinco estudos a serem incluídos e os dados conflitantes serão resolvidos por consenso e, se necessário, um terceiro revisor poderá arbitrar. Após essa etapa, se necessário, o formulário será ajustado, para extração definitiva das informações de cada estudo e esse processo relatado no momento da apresentação dos resultados da revisão, que será por meio do relatório final, que será publicado em formato de artigo. 
Quadro 2. Formulário para extração de dados dos artigos selecionados.

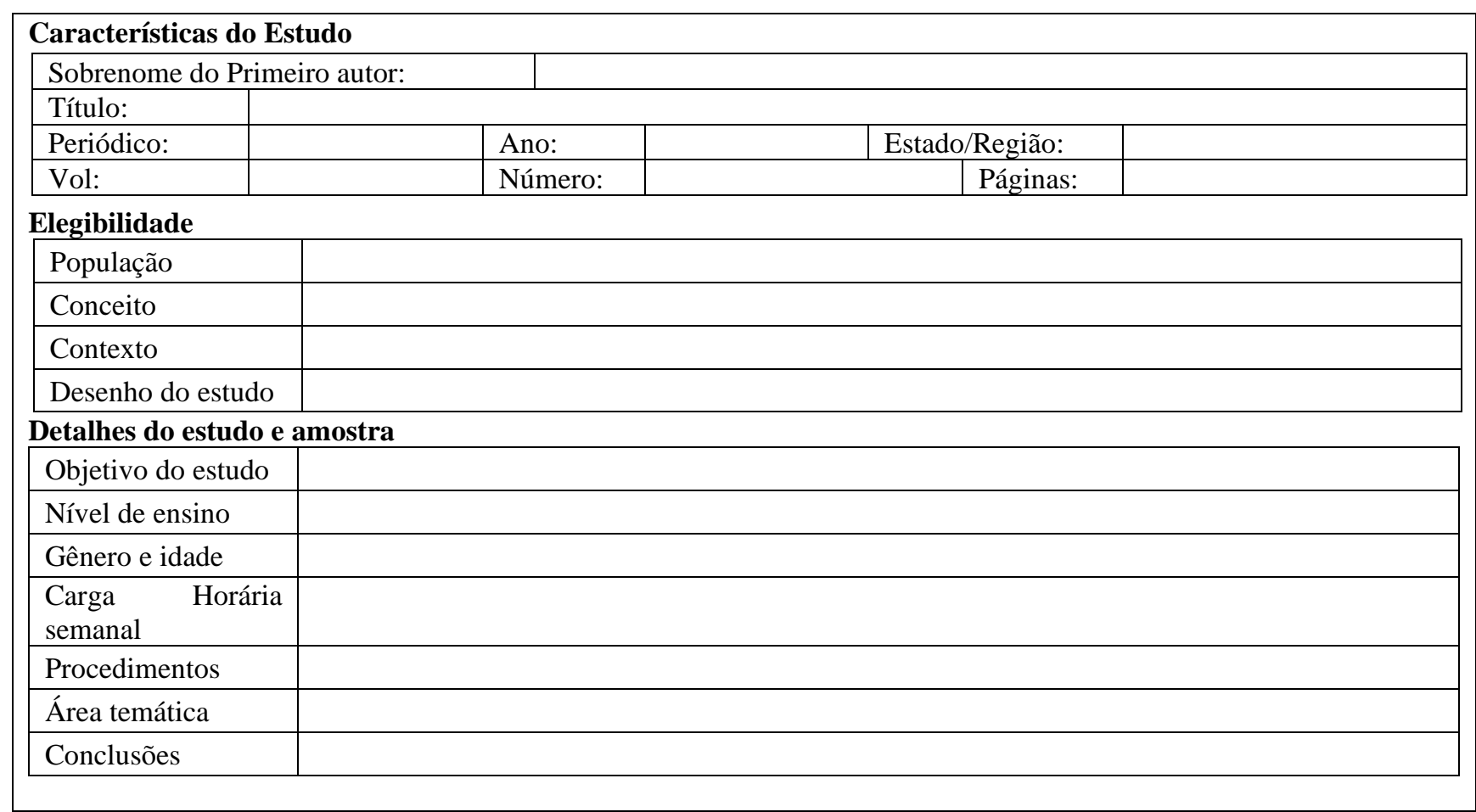

Fonte: Autores.

\subsection{Sumarização e apresentação dos dados.}

Os dados extraídos serão apresentados por meio de tabelas, quadros e gráficos, alinhados aos objetivos e questões desta revisão de escopo. Os gráficos reportarão a distribuição de estudos quanto ao ano, distribuição de produção por periódicos, local de desenvolvimento das pesquisas e tipos de estudos. A estatística descritiva por meio de frequências absolutas e relativas, serão consideradas para descrever e caracterizar variáveis como categoria temática e número de publicações por região. As tabelas apresentarão uma síntese dos estudos por autor (ano) e região de publicação, objetivo do artigo, amostra e temática.

Um resumo narrativo acompanhará os resultados mapeados e descreverão como os resultados se relacionam com o objetivo da revisão e perguntas. Ao analisar os estudos incluídos, esses serão agrupados por categorias temáticas, conforme número de ocorrência das mesmas, que serão analisadas e apresentadas de forma descritiva e considerando a técnica de análise de conteúdo. E, por se tratar de uma revisão de escopo, a avaliação da qualidade metodológica dos estudos incluídos não será realizada.

\section{Resultados esperados}

Há uma robusta literatura relacionada à voz do professor são inúmeras na Fonoaudiologia, sendo voltadas tanto para a promoção da saúde vocal quanto para a descoberta de estratégias eficazes para garantir ao professor um bom desempenho de suas atividades profissionais. O professor necessita de uma voz que seja capaz de transmitir o conteúdo de forma eficiente e estabelecer uma boa comunicação com o estudante. Uma busca inicial na literatura apontou para a existência de diversos estudos primários, nacionais e internacionais, que buscaram temáticas diversas sobre a problemática vocal do professor na Fonoaudiologia. 
A diversidade de estudos e a busca pela compreensão da distribuição destes, ao longo dos anos, na Fonoaudiologia brasileira, permitirá um conhecimento amplo sobre como tem sido conduzidas as pesquisas na área. Conhecer como vem sendo realizado os estudos primários permite compreender o impacto das abordagens junto aos professores, seja na prevenção ou na intervenção/assistência. Assim, por meio de um mapeamento transparente e sistemático pode-se compreender o perfil das publicações nacionais, publicadas em periódicos da Fonoaudiologia, e planejar outros estudos, preenchendo as lacunas que ainda precisam ser mais investigadas na Fonoaudiologia quando se trata de voz do professor.

Pretende-se, assim, com o desenvolvimento do estudo aqui planejado, conhecer o cenário das publicações em periódicos nacionais de Fonoaudiologia e planejar estratégias futuras de intervenção ou ações de promoção à saúde e bem-estar vocal do professor, seja ele do ensino infantil, fundamental, médio ou superior.

\section{Considerações Finais}

O estudo sobre a problemática vocal do professor recebe destaque na área da voz, dentro da Fonoaudiologia. Esse profissional quando tem seu instrumento de trabalho comprometida, a voz, pode ter sua atividade laboral prejudicada. Além do mais, a voz é um recurso comunicativo importante e representa o sujeito, podendo uma alteração comprometer a interação social e transmissão do conteúdo/aprendizagem do aluno em sala de aula. Um problema vocal pode, também, trazer consequências no aspecto emocional desse docente, assim como predisposição às alterações vocais.

Compreender as pesquisas desenvolvidas, por meio do mapeamento aqui proposto, e publicizar seus resultados, permitirá aos profissionais que trabalham na área da voz do professor um conhecimento do cenário atualizado. O que permitirá a projeção de estudos futuros e uma prática apoiada em evidências.

\section{Contribuições dos autores}

EPGM, VFAP e LVDC desenharam o protocolo. MAS e EPGM selecionarão os estudos, a extração dos dados será realizada por, MAS e LVDC, com supervisão de VFAP. A escrita final do protocolo foi realizada por EPGM, VFAP e MAS, com contribuições de LVDC.

\section{Conflitos de interesse}

Os autores declaram não apresentar nenhum tipo de conflito de interesse.

\section{Referências}

Araújo, A. N. B. de, Gomes, A. de O. C., Miranda, D. P. de, Silva, C. L. P. da, Lira, Z. S. de, \& Lucena, J. A. (2015). Programa de promoção à saúde vocal do Servidor Público da Universidade Federal de Pernambuco. In L. P. Ferreira, M. A. de A. \& Silva, S. P. P. Giannini (Eds.), Distúrbio de voz relacionado ao trabalho: práticas fonoaudiológicas. 73-81. Roca.

Arksey, H., \& O’Malley, L. (2005). Scoping studies: towards a methodological framework. International Journal of Social Research Methodology, 8(1), 1932. https://doi.org/10.1080/1364557032000119616

Behlau, M., Azevedo, R., \& Pontes, P. (2001). Conceito de voz normal e disfonia. In M. Behlau (Ed.), Voz: O livro do especialista, 53-79. Editora Revinter.

Behlau, M., Zambon, F., Guerrieri, A. C., \& Roy, N. (2012). Epidemiology of Voice Disorders in Teachers and Nonteachers in Brazil: prevalence and adverse effects. Journal of Voice, 26(5), 665.e9-665.e18. https://doi.org/10.1016/j.jvoice.2011.09.010

Caporossi, C., \& Ferreira, L. P. (2010). Sintomas vocais e fatores relativos ao estilo de vida em professores. Revista CEFAC, 13(1), 132-139. https://doi.org/10.1590/S1516-18462010005000099

Dragone, M. L. S., Ferreira, L. P., Giannini, S. P. P., Simões-Zenari, M., Vieira, V. P., \& Behlau, M. (2010). Voz do professor: uma revisão de 15 anos de contribuição fonoaudiológica. Revista da Sociedade Brasileira de Fonoaudiologia, 15(2), 289-296. https://doi.org/10.1590/S1516-80342010000200023

Evangelista, R. A., Loureiro, H. M. A. M., Silva, L. A., \& Mendes, A. M. O. da C. (2019). Programas de promoción de la salud ocupacional implementados en profesores de instituciones de educación superior. Enfermería Actual de Costa Rica, 37, 263-272. https://doi.org/10.15517/revenf.v0ino.37.36326 
Research, Society and Development, v. 10, n. 10, e314101018872, 2021

(CC BY 4.0) | ISSN 2525-3409 | DOI: http://dx.doi.org/10.33448/rsd-v10i10.18872

Ferreira, L. P., Giannini, S. P. P., Alves, N. L. L., Brito, A. F. de, Andrade, B. M. R. de, \& Latorre, M. do R. D. de O. (2016). Distúrbio de voz e trabalho docente. Revista CEFAC, 18(4), 932-940. https://doi.org/10.1590/1982-0216201618423915

Fillis, M. M. A., Andrade, S. M. de, González, A. D., Melanda, F. N., \& Mesas, A. E. (2016). Frequência de problemas vocais autorreferidos e fatores ocupacionais associados em professores da educação básica de Londrina, Paraná, Brasil. Cadernos de Saúde Pública, 32(1), e00026015. https://doi.org/10.1590/0102-311X00026015

Giannini, S. P. P., Latorre, M. do R. D. de O., \& Ferreira, L. P. (2012). Distúrbio de voz e estresse no trabalho docente: um estudo caso-controle. Cadernos de Saúde Pública, 28(11), 2115-2124. https://doi.org/10.1590/S0102-311X2012001100011

Gomes, N. R., Medeiros, A. M. de, \& Teixeira, L. C. (2016). Autopercepção das condições de trabalho por professores de ensino fundamental. Revista CEFAC, 18(1), 167-173. https://doi.org/10.1590/1982-021620161819515

Morais, E. P. G., Azevedo, R. R., \& Chiari, B. M., et al. (2012). Correlação entre voz, autoavaliação vocal e qualidade de vida em voz de professoras. Revista CEFAC, 14(5), 892-900. https://doi.org/10.1590/S1516-18462012005000032

Mortimer, E. F. (2014). Recursos de expressividade usados por uma professora universitária. Distúrbios da Comunicação, 26(4), 777-789.

Peters, M., Godfrey, C., McInerney, P., Munn, Z., Trico, A., \& Khalil, H. (2020). Chapter 11: Scoping Reviews (2020 version). In Aromataris, E., \& Munn, Z. (Eds.), JBI Manual for Evidence Synthesis. JBI. https://doi.org/10.46658/JBIMES-20-12

Ramos, J. S., Feniman, M. R., Gielow, I., \& Silverio, K. C. A. (2018). Correlation between Voice and Auditory Processing. Journal of Voice, 32(6), 771.e25771.e36. https://doi.org/10.1016/j.jvoice.2017.08.011

Santos, T. D. dos, \& Andrada e Silva, M. A. de. (2016). Comunicação não verbal com profissionais da voz: o que se pesquisa na fonoaudiologia. Revista CEFAC, 18(6), 1447-1455. https://doi.org/10.1590/1982-021620161865116

Schwartz, S. R., Cohen, S. M., Dailey, S. H., Rosenfeld, R. M., Deutsch, E. S., Gillespie, M. B., Granieri, E., Hapner, E. R., Kimball, C. E., Krouse, H. J., McMurray, J. S., Medina, S., O’Brien, K., Ouellette, D. R., Messinger-Rapport, B. J., Stachler, R. J., Strode, S., Thompson, D. M., Stemple, J. C., ... Patel, M. M. (2009). Clinical Practice Guideline: Hoarseness (Dysphonia). Otolaryngology-Head and Neck Surgery, 141(1_suppl), 1-31. https://doi.org/10.1016/j.otohns.2009.06.744

Tricco, A. C., Lillie, E., Zarin, W., O’Brien, K. K., Colquhoun, H., Levac, D., Moher, D., Peters, M. D. J., Horsley, T., Weeks, L., Hempel, S., Akl, E. A., Chang, C., McGowan, J., Stewart, L., Hartling, L., Aldcroft, A., Wilson, M. G., Garritty, C., ... Straus, S. E. (2018). PRISMA Extension for Scoping Reviews (PRISMA-ScR): checklist and explanation. Annals of Internal Medicine, 169(7), 467-473. https://doi.org/10.7326/M18-0850

Valente, A. M. S. L., Botelho, C., \& Silva, A. M. C. da. (2015). Distúrbio de voz e fatores associados em professores da rede pública. Revista Brasileira de Saúde Ocupacional, 40(132), 183-195. https://doi.org/10.1590/0303-7657000093814

Vieira, V. P. (2009). Tratamento dos distúrbios da voz baseado em evidências. Diagnóstico e tratamento, 14(1), 19-21. http://files.bvs.br/upload/S/14139979/2009/v14n1/a0005.pdf 